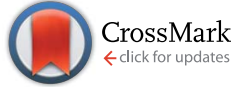

Cite this: RSC Adv., 2015, 5, 17149

\title{
Correction: Towards applications in catalysis: investigation on photocatalytic activities of a derivative family of the Keplerate type molybdenum-oxide based polyoxometalates
}

\author{
Yunshan Zhou, ${ }^{\star}$ Libo Qin, Chao Yu, Tian Xiong, Lijuan Zhang, ${ }^{*}$ Waqar Ahmad \\ and Hao Han
}

DOI: $10.1039 / \mathrm{c} 5 \mathrm{ra90010 \textrm {g }}$

Correction for 'Towards applications in catalysis: investigation on photocatalytic activities of a derivative family of the Keplerate type molybdenum-oxide based polyoxometalates' by Yunshan Zhou et al., RSC www.rsc.org/advances Adv., 2014, 4, 54928-54935.

The authors wish to clarify a few sentences by stating that $\mathrm{Mo}_{132}$ anions are unstable in solution at very low concentrations. Therefore, the phrase "at very low concentrations" has been added to the following sections of the article:

The second sentence of the Abstract on page 54928 should read: "The $\mathrm{Mo}_{132}$ anions are found to be photocatalytically active centers, however they are unstable and subjected to decomposition in the solution form at very low concentrations".

In the tenth line of the first paragraph of the right column on page 54931, the correct sentence is "However, the water-soluble compounds 1 and 3 were naturally unstable in the solution at very low concentrations and could be decomposed completely within a certain period of time, this means that the Keplerate type $\left\{\mathrm{Mo}_{132}\right\}$ anions need to be stabilized and protected in aqueous solution".

In the fifth line of the Conclusions section on page 54935, the correct sentence is "The $\mathrm{Mo}_{132}$ anions were found to be the active centers under UV irradiation to degrade RhB, while they were unstable in solution at very low concentrations and need to be protected by organic cations endowing stability of the anions due to the resultant water-tolerant property and the hydrophobic interactions between the alkyl chains of cations in the hybrid materials in a solid state".

The authors apologise for these errors and are indebted to Prof. Dr Achim Müller (Fakultät für Chemie, Universität Bielefeld, Germany) for the exchange of information and the discussion regarding this corrigendum.

The Royal Society of Chemistry apologises for these errors and any consequent inconvenience to authors and readers. 